\title{
Desarrollo de hot-dog a base de carne de pollo (Gallus gallus) mediante la Ingeniería Kansei Tipo II
}

\author{
Development of hot-dog based on chicken (Gallus gallus) by Type II Kansei Engineering
}

\author{
Gustavo Gavino Puma Isuiza ${ }^{1 *}$ y Carlos Núñez Saavedra²
}

* Autor de correspondencia

\section{Resumen}

El objetivo de la presente investigación se fue aplicar la metodología Kansei tipo II, para el desarrollo de hot-dog altamente emocional. La elección del dominio se realizó basándonos en un estudio de mercado de embutidos en Lima Metropolitana, habiendo elegido al hot-dog de pollo entre hot-dog de pavo, chorizo y morcilla. Para la reducción del espacio semántico se aplicó un método mixto, obteniendo $15 \mathrm{Kansei}$ (8 elementos sensoriales y 7 expresiones hedónicas). El espacio de propiedades (tipo de funda y color del hot-dog) fue determinado mediante el modelo Kano. Los prototipos se elaboraron siguiendo las siguientes operaciones: molienda, mezclado, cutterizado, embutido, escaldado, enfriado y escurrido. En la etapa de síntesis se realizó la Teoría de Cuantificación 1 (QT1), donde las variables regresoras fueron las categorías del espacio de propiedades y las variables respuesta fueron las valoraciones promedio de los Kansei. La aplicación de Ingeniería Kansei tipo II permitió establecer la relación entre las necesidades afectivas del consumidor y el espacio de propiedades obtenido mediante el modelo Kano en el desarrollo de hot-dog de carne de pollo.

Palabras clave: modelo Kano; regresión QT1; altamente emocional; análisis de componentes principales; análisis de conglomerados.

\begin{abstract}
The objective of the present investigation was to apply the Kansei type II methodology, for the development of hotdog highly emotional. The choice of domain was based on a market study of sausages in Metropolitan Lima, having chosen the chicken hot-dog among turkey hot-dog, sausage and blood sausage. For the reduction of semantic space a mixed method was applied, obtaining $15 \mathrm{Kansei}$ ( 8 sensorial elements and 7 hedonic expressions). The property space (type of sheath and color of the hot dog) was determined using the Kano model. The prototypes were elaborated by the following operations: grinding, mixing, cutterized, sausage, scald, cooled and drained. In the synthesis stage, the QT1 regression was performed, where the regressor variables were the property space, categories and the response variables were the average values of the Kansei. The application of Engineering Kansei type II allowed to establish the relationship between the affective needs of the consumer and the space of properties obtained by the Kano model in the development of chicken hot-dog.
\end{abstract}

Keywords: Kano model; QT1 regression; Highly emotional; principal component analysis; cluster analysis.

\section{Introducción}

El hot-dog es un embutido escaldado constituido por una masa hecha de carne magra y grasa de porcino, que puede tener carne de bovino, cerdo, caprino, equino, etc. y verduras; las cuales deben estar trituradas y mezcladas. Además puede o no tener agregados de harinas, féculas, almidones o especias que deben estar distribuidos uniformemente (Elías et al., 2000).

La Ingeniería Kansei (IK) es una metodología de desarrollo de productos orientada al consumidor, creada en Japón en los años 80 por Mitsuo Nagamachi. Establece procedimientos para traducir y trasladar las percepciones emocionales, gustos y sensaciones de los consumidores, generados por productos existentes o conceptos, en parámetros de diseño; logrando incorporar al producto los valores emocionales que atraen al consumidor de forma cuantitativa (Nagamachi, 1994 y 2011). La IK se aplica recientemente al diseño de productos alimentarios. No es ampliamente usada en el Perú, debido a la poca comprensión de su procedimiento y aplicación metodológica.

Kansei es una palabra japonesa que se define como la impresión que tiene una persona de un determinado producto, entorno o situación, utilizando sus cinco sentidos, que es procesada y basada en sus vivencias, experiencias y conocimientos, provocando un sentimiento (consciente 
o inconsciente) que se traduce en imágenes o impresiones subjetivas (Schütte, 2005; Hirata, 2009; Nagamachi, 2011; Marco-Almagro, 2011).

Hasta la actualidad se han desarrollado, probado y testeado seis metodologías o tipos (I, II, III, IV, V y VI) que se diferencian uno de otros principalmente por la forma de presentación del producto, el análisis estadístico y la construcción de modelos matemáticos predictivos. La IK tipo II es la metodología más utilizada actualmente; se trata de una técnica especialmente centrada en el usuario, que tiene en cuenta todos los aspectos emocionales y sensitivos de los futuros consumidores. Es una metodología, basada en trasladar y plasmar las imágenes mentales, percepciones, sensaciones y gustos del consumidor en los elementos de diseño que componen un producto (Álvarez, 2011; Nagamachi, 2011; Nagamachi y Mohd, 2011).

En el contexto de la integración de los sentimientos en los productos, el modelo Kano es de gran relevancia. Este modelo permite identificar las características de encantamiento que son de gran importancia para que el nuevo producto se introduzca con éxito en el mercado ya que satisfacen necesidades latentes y profundas del consumidor (Hirata, 2009; Schütte, 2002; Schütte, 2005).

Por lo expuesto, el objetivo principal de la investigación fue aplicar la metodología Kansei Tipo II para desarrollar hot-dog altamente emocional a base de carne de pollo (Gallus gallus). Así mismo, determinar las necesidades Kansei de dos sectores socio-económicos del mercado de Lima Metropolitana (sectores B y C).

\section{Materiales y métodos}

Lugar de Ejecución, La investigación se realizó en la Planta Piloto de Alimentos y el Laboratorio de Evaluación Sensorial, pertenecientes a la Facultad de Industrias Alimentarias de la Universidad Nacional Agraria La Molina, Lima-Perú.

Materia prima, La materia prima principal fue pechuga de pollo (Gallus gallus).

Metodología experimental, Para la aplicación de la metodología Kansei Tipo II, se siguió el procedimiento establecido por Marco-Almagro (2011) (Figura 1) y los lineamientos establecidos por Marco-Almagro y Schütte (2014) y Puma (2017).

\section{Selección del dominio}

Se eligió el producto en estudio y se definió el público objetivo al que se dirigió.

\section{Determinación del espacio semántico Espacio semántico inicial}

Para establecer la lista inicial de palabras Kansei se efectuó un sondeo de opinión con el público objetivo a los cuales

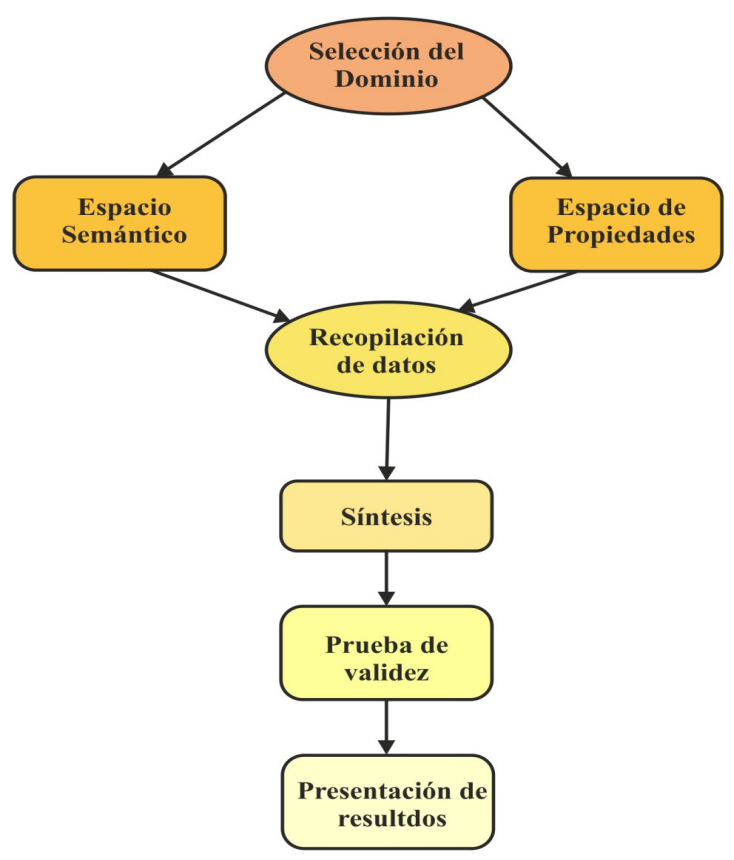

Figura 1. Modelo General de Ingeniería Kansei Fuente: Marco-Almagro, 2011

se les permitió interactuar con el alimento antes de ser consumido; además, dar su apreciación después de haber evaluado el producto.

\section{Espacio semántico reducido}

En primer lugar, se eliminaron los Kansei redundantes y los que no estaban relacionados con el objetivo de la investigación. En segundo lugar, se separaron los Kansei en expresiones hedónicas y atributos sensoriales. En tercer lugar, se realizó un primer Diagrama de Afinidad (DA) con los Kansei que quedaron en la lista. En cuarto lugar, se realizó una evaluación sensorial afectiva (Marco-Almagro y Schütte, 2014). En quinto lugar, se realizó un segundo DA con los Kansei del primer DA. Por último, se efectuó el Análisis Cluster no jerárquico de $k$-medias y el Análisis de Componentes Principales.

\section{Espacio Semántico Final}

Se elaboró la ficha de evaluación final. Para ello, se tomó en cuenta lo sugerido por Nagamachi (2011) y MarcoAlmagro (2011) en cuanto a la polarización de las palabras Kansei y la escala de siete puntos.

\section{Espacio de propiedades}

Se desarrolló en tres etapas. En la Etapa I, se eligieron las características que los consumidores percibían al interactuar con los productos. En la Etapa II se desarrolló el cuestionario Kano. En la etapa III, se determinaron las características de encantamiento, con las cuales se elaboraron los prototipos de hot-dog. 


\section{Elaboración de hot-dog}

Para la elaboración de los prototipos de hot-dog se siguió el procedimiento mostrado en la Figura 2, que se detalla a continuación:

Molienda: la pechuga de pollo y la grasa dorsal de cerdo fueron molidas (Mainca ${ }^{\circledR}$, PM-70) a través de un disco de $3 \mathrm{~mm}$ de diámetro.

Cutterizado: se agregó al cutter $\left(\right.$ Hobart $\left.^{\circledR}, 84145\right)$ los insumos en el siguiente orden: pechuga de pollo molida, sal $\left(\right.$ Emsal $^{\circledR}$ ), sal de cura al 20\% (Alitecno ${ }^{\circledR}$ ), eritorbato, tripolifosfato de sodio (Alitecno ${ }^{\circledR}$ ), mitad del hielo, grasa dorsal de cerdo, el resto de hielo, pimienta, nuez moscada y comino esterilizados $\left(\mathrm{GLM}^{\circledR}\right)$, y color carmín natural $\left(\right.$ Frutarom $\left.^{\circledR}\right)$.

Embutido: cuando la masa estuvo homogénea se embutió $\left(\right.$ Boxa $\left.^{\circledR}, \mathrm{MQE0}\right)$, tratando de no introducir aire en las fundas no comestibles (Alitecno ${ }^{\circledR}$ ) y fundas comestibles $\left(\right.$ QuimSa $^{\circledR}$ )

Escaldado: se escaldó en una olla de acero inoxidable (20 $\mathrm{L}$ de capacidad) con agua entre $75^{\circ} \mathrm{C}-80^{\circ} \mathrm{C}$, hasta que la temperatura interna del producto alcanzó entre $68^{\circ} \mathrm{C}-72$ ${ }^{\circ} \mathrm{C}$.

Enfriado: se enfriaron los productos en agua helada (15 ${ }^{\circ} \mathrm{C}$ ) hasta que la temperatura en el punto más frío fue menor a $25^{\circ} \mathrm{C}$.

Escurrido: se dejó escurrir el agua del hot-dog con ayuda de un colador.

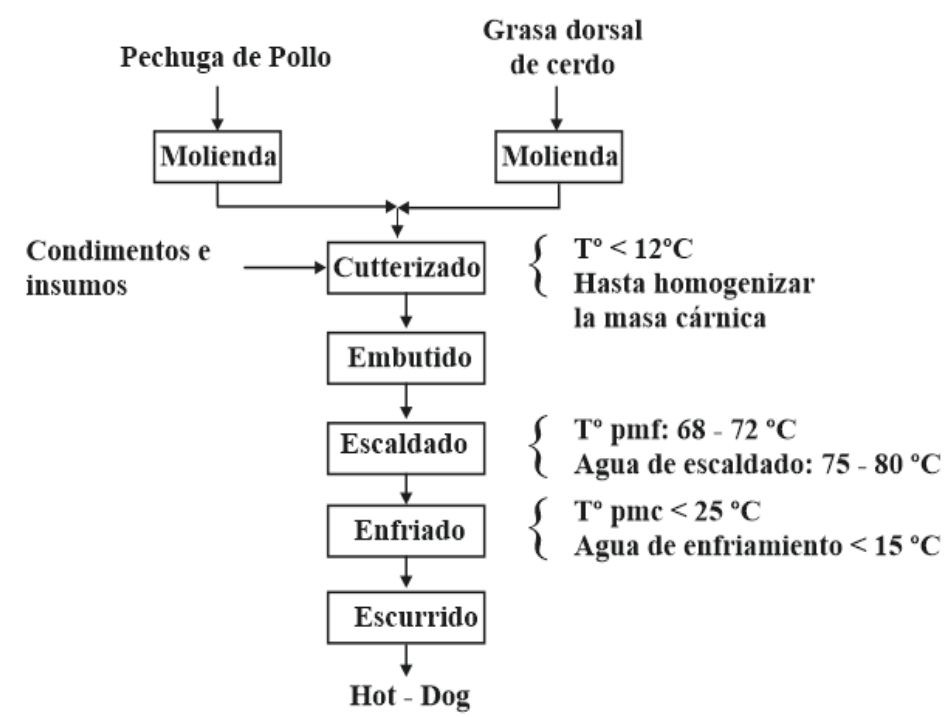

Figura 2. Flujo de operaciones para la obtención de hot-dog a base de carne de pollo

\section{Síntesis}

Se realizó la regresión QT1 para relacionar el espacio semántico reducido (palabras Kansei) con el espacio de propiedades (categorías).

\section{Análisis estadísticos}

El análisis de conglomerados de k-medias y el análisis de componentes principales se ejecutó con el software SPSS ${ }^{\circledR}$
Versión 21 y la regresión QT1 con el software $\mathrm{R}^{\circledR}$ versión 3.1.1.

\section{Análisis de Conglomerados no jerárquico de $\boldsymbol{k}$-medias}

Se realizó un Análisis de clúster de $k$-medias a las expresiones hedónicas y a los elementos sensoriales, por separado, para reducir la lista inicial de palabras Kansei generadas por los consumidores (Marco-Almagro, 2011; Meullenet et al., 2007).

\section{Análisis de Componentes Principales (ACP)}

Se realizó el ACP a las expresiones hedónicas y los elementos sensoriales, para su mejor visualización (Ezequiel y Aldas, 2005; Johnson y Wichern, 2007).

\section{Teoría de cuantificación 1 (QT1)}

Se realizó la regresión QT1 considerando a las categorías del espacio de propiedades como las variables regresoras y los Kansei como variables respuestas (Marco-Almagro, 2011; Damodar, 2005; Marco-Almagro y Schütte, 2014; Álvarez, 2009).

\section{Resultados y discusión}

\section{Selección del Dominio}

El producto en estudio (hot-dog de pollo) fue elegido entre los embutidos presentes en el mercado de Lima Metropolitana: hot-dog de pollo, hot-dog de pavo, chorizo y morcilla. Se eligió hot-dog a base de carne de pollo (Gallus gallus) debido a que es el producto que se consume más en los sectores socioeconómicos $\mathrm{B}$ y $\mathrm{C}$ de Lima MetropolitanaM, los cuales ocupan el 64,1\% de la población (APEIM, 2016).

Para la determinación del espacio semántico reducido se utilizó hot-dog de pollo de las marcas Otto Kunz, Razzeto y Braedt; ya que éstas son las que ocupan alrededor del $80 \%$ del mercado de embutidos en el sector B y C de LM (Palazuelos y Blázquez, 2013). Estos tres hot-dog fueron extraídos de un total de seis marcas (Braedt, Laive, Otto Kunz, Salchichería Alemana, Razzeto y San Fernando); debido a que poseen características sensoriales diferentes, tanto el hot-dog Otto Kunz, Razzeto y Braedt, tiene diferente coloración superficial, tamaño y calibre; la marca Razzeto no tiene funda comestible, a diferencia de las marcas Braedt y Otto Kunz; estas dos últimas se diferencian en que la primera es ahumada. Marco-Almagro (2011) recomienda usar productos que incluyan características diferenciadas para así obtener una base de datos Kansei representativa.

\section{Determinación del espacio semántico final}

La lista inicial del espacio semántico fue generada por 30 panelistas (16 hombres y 14 mujeres) de 20 a 35 
años de edad de los sectores B y C de LM. Además, se consideraron las expresiones: innovador y lo probaría otra vez, de la investigación de Marco-Almagro y Schütte (2014) quienes trabajaron con cañitas de carne. No fue necesario incluir los Kansei de propagandas de publicidad, ya que estas (tradicional, sabroso y rico) fueron generadas por los consumidores. Marco-Almagro y Schütte (2014) mencionan que en la elaboración de la lista inicial se pueden incluir palabras Kansei de revistas, sitios web, consumidores, expertos o estudios de Ingeniería Kansei (IK) relacionados.

Cada panelista generó entre 11 a 20 palabras Kansei haciendo un total de 365 expresiones. De la base de datos inicial se eliminaron aquellos Kansei que eran variantes de expresiones como: color débil, colorido, textura suave, textura rígida, envoltura dura, parte externa dura, cubierta dura, etc. o eran irrelevantes para el estudio, como: lechoso, descompuesto, oxidado y buena emulsión; ya que ésta última fue considerada específica del producto. MarcoAlmagro (2011) menciona que en esta fase se pueden eliminar términos que son muy especializados o que están fuera del contexto de la investigación. Nagamachi (2011) y Nagamachi y Mohd (2011) mencionan la problemática de incluir Kansei negativos ya que hasta la actualidad no se ha establecido procedimientos para su inclusión en la base de datos, por lo que recomiendan retirarlos de la lista inicial.

Después de eliminar los Kansei redundantes e irrelevantes quedaron 102 palabras Kansei (59 elementos sensoriales y 43 expresiones hedónicas); cantidad que se encuentra entre 100 a $600 \mathrm{Kansei}$, recomendado por Nagamachi y Mohd (2011) quienes además mencionan que un número inferior a 100 no inhabilita realizar un estudio de IK. Hirata (2009) realizó investigaciones de diseño de envases de cerveza con 92 palabras Kansei generadas por el estudio de Hishihara. Marco-Almagro (2011) en su estudio de diseño de un jugo altamente emocional reportó 123 palabras Kansei y, Marco-Almagro y Shútte (2014) recogieron un total de 151 expresiones, de las cuales después de eliminar las redundantes e irrelevantes quedaron 55 expresiones hedónicas y 32 elementos sensoriales, haciendo un total de 87 Kansei.

En la Tabla 1 y 2 se presentan el resumen del análisis mixto efectuado a los 102 Kansei para las expresiones hedónicas y los elementos sensoriales, respectivamente. En el primer Diagrama de Afinidad (DA), se agruparon los Kansei semejantes; por ejemplo, a la sierra, receta tradicional, casero y hecho a leña formaron el Kansei Tradicional; del mismo modo se procedió con los demás Kansei. Luego del primer DA quedaron 42 palabras Kansei (23 elementos sensoriales y 19 expresiones hedónicas), las cuales fueron sometidas nuevamente a un DA, prosiguiendo del mismo modo que para el primer DA, quedando 15 palabras Kansei de nivel superior (siete expresiones hedónicas y ocho elementos sensoriales, indicados por el superíndice en la Tabla 1 y 2).

El segundo DA determinó la cantidad de grupos que se establecieron para realizar el Análisis Clúster de $k$-medias, que para el caso de los elementos sensoriales fue ocho y para las expresiones hedónicas fue siete. Marco-Almagro (2011) menciona que en la agrupación no jerárquica de $k$-medias, los grupos obtenidos a partir del DA se pueden utilizar como grupos iniciales para el análisis, refinando así la reducción con los datos recogidos.

Tabla 1. Clasificación de las expresiones hedónicas antes del Análisis Clúster (segundo diagrama de afinidad) y después del análisis clúster no jerárquico por $k$-medias.

\begin{tabular}{|c|c|}
\hline $\begin{array}{l}\text { Expresiones hedónicas antes del } \\
\text { análisis clúster* }\end{array}$ & $\begin{array}{l}\text { Expresiones hedónicas } \\
\text { después del análisis clúster** }\end{array}$ \\
\hline Tradicional $^{1}$ & Tradicional $^{1}$ \\
\hline Clásico ${ }^{1}$ & Clásico ${ }^{1}$ \\
\hline Saludable $^{2}$ & Saludable $^{2}$ \\
\hline Natural $^{2}$ & Natural $^{2}$ \\
\hline Nutritivo $^{2}$ & Nutritivo $^{3}$ \\
\hline Llamativo $^{3}$ & Calidad $^{3}$ \\
\hline Peculiar $^{3}$ & Bueno $^{3}$ \\
\hline Emocionante $^{3}$ & Brinda confianza ${ }^{3}$ \\
\hline Innovador $^{3}$ & Lo consumiría $^{3}$ \\
\hline Calidad $^{4}$ & Llamativo $^{4}$ \\
\hline Bueno $^{4}$ & Peculiar $^{4}$ \\
\hline Brinda confianza ${ }^{4}$ & Emocionante $^{5}$ \\
\hline Placentero $^{5}$ & Innovador 5 \\
\hline Sabroso $^{5}$ & Placentero ${ }^{6}$ \\
\hline Probaría otra vez ${ }^{5}$ & Sabroso ${ }^{6}$ \\
\hline Lo compraría $^{6}$ & Probaría otra vez ${ }^{6}$ \\
\hline Lo consumiría ${ }^{6}$ & Lo compraría ${ }^{6}$ \\
\hline Listo para comer ${ }^{6}$ & Me gusta ${ }^{6}$ \\
\hline Me gusta ${ }^{7}$ & Listo para comer ${ }^{7}$ \\
\hline
\end{tabular}

El segundo DA determinó la cantidad de grupos que se establecieron para realizar el Análisis Clúster de $k$-medias, que para el caso de los elementos sensoriales fue ocho y para las expresiones hedónicas fue siete. Marco-Almagro (2011) menciona que en la agrupación no jerárquica de $k$-medias, los grupos obtenidos a partir del DA se pueden utilizar como grupos iniciales para el análisis, refinando así la reducción con los datos recogidos. Nagamachi (2011) y Nagamachi y Mohd (2011) realizaron investigaciones con análisis de conglomerados y análisis factorial; Hirata (2009) optó por utilizar el análisis de componentes principales.

En la primera columna de la Tabla 1 se muestran las 19 expresiones hedónicas, cada una tiene un número del uno a siete que da la asignación inicial a un clúster. Después del procedimiento de $k$-medias, se observa en la segunda columna que solamente el clúster que contenía el Kansei "tradicional" se mantiene sin cambio. Los demás grupos se modificaron en tamaño; algunos contienen más Kansei y otros menos, por ejemplo, el grupo dos que inicialmente tenía tres Kansei (saludable, natural y nutritivo), luego de realizar el análisis clúster de k-medias se redujo a dos Kansei (saludable y natural). En otros casos se conservó el 
mismo tamaño pero diferente Kansei, como en el caso del grupo siete en dónde el Kansei "me gusta" fue cambiado por el Kansei "Listo para comer".

Tabla 2. Clasificación de los elementos sensoriales antes del Análisis Clúster (segundo diagrama de afinidad) y después del análisis clúster no jerárquico por $k$-medias

\begin{tabular}{|c|c|}
\hline $\begin{array}{c}\text { Elementos sensoriales antes del } \\
\text { análisis clúster* }\end{array}$ & $\begin{array}{c}\text { Elementos sensoriales después } \\
\text { del análisis clúster** }\end{array}$ \\
\hline Color uniforme $^{1}$ & Color uniforme $^{1}$ \\
\hline Colorido $^{1}$ & Colorido $^{1}$ \\
\hline Textura uniforme ${ }^{2}$ & Textura uniforme $^{2}$ \\
\hline Textura plástica ${ }^{2}$ & Forma simétrica ${ }^{2}$ \\
\hline Textura externa dura ${ }^{2}$ & Tamaño adecuado ${ }^{2}$ \\
\hline Duro $^{2}$ & Sabor a pollo ${ }^{3}$ \\
\hline Sabor a pollo ${ }^{3}$ & Sabor intenso ${ }^{3}$ \\
\hline Sabor intenso ${ }^{3}$ & Sabor definido ${ }^{3}$ \\
\hline Sabor definido ${ }^{3}$ & Sabor a colorante ${ }^{3}$ \\
\hline Sabor a colorante ${ }^{3}$ & Textura plástica ${ }^{4}$ \\
\hline Aroma sintético ${ }^{3}$ & Aroma sintético ${ }^{4}$ \\
\hline Olor fuerte ${ }^{4}$ & Brilloso $^{4}$ \\
\hline Aroma duradero ${ }^{4}$ & Olor fuerte ${ }^{5}$ \\
\hline Brilloso $^{5}$ & Aroma duradero ${ }^{5}$ \\
\hline Oscuro $^{5}$ & Condimentado $^{5}$ \\
\hline Ahumado $^{5}$ & Con colorante ${ }^{5}$ \\
\hline Áspero al masticar ${ }^{6}$ & Textura externa dura ${ }^{6}$ \\
\hline Grumoso ${ }^{6}$ & Duro $^{6}$ \\
\hline Forma simétrica $^{7}$ & Áspero al masticar ${ }^{6}$ \\
\hline Tamaño adecuado $^{7}$ & Grumoso $^{6}$ \\
\hline Condimentado $^{8}$ & Oscuro $^{7}$ \\
\hline Con colorante ${ }^{8}$ & Ahumado ${ }^{7}$ \\
\hline Salado ${ }^{8}$ & Salado $^{8}$ \\
\hline
\end{tabular}

En la primera y segunda columna de la Tabla 2 se visualiza que, al igual que en el caso de las expresiones hedónicas, algunos grupos formados por el DA fueron reordenados al realizar el análisis de conglomerados de k-medias. Solamente el clúster que contenía los Kansei "Color uniforme" y "Colorido" se mantuvo sin cambio. Los demás grupos se modificaron en tamaño, por ejemplo, el grupo dos que inicialmente tenía cuatro Kansei (textura uniforme, textura plástica, textura externa dura y duro), luego de realizar el análisis clúster de k-medias se redujo a tres Kansei (textura uniforme, forma simétrica y tamaño adecuado). Marco-Almagro (2011) en su estudio de Ingeniería Kansei aplicado al desarrollo de un jugo altamente emocional, también encontró una reorganización de los Kansei generados en el primer Diagrama de Afinidad; además menciona que los grupos finales pueden ser considerados, tan lógicos como los iniciales, porque tienen la ventaja de incorporar información extraída de evaluaciones con el producto en estudio.

En la Figura 3 se observa que las marcas Braedt, Razzeto y Otto Kunz se encuentran distribuidas en el primer, segundo y cuarto cuadrante, respectivamente; esto quiere decir que las tres marcas tienen diferencias en cuanto a las 19 expresiones hedónicas evaluadas (Varela y Ares, 2014). Este resultado es coherente, ya que para los estudios de IK, se evalúan muestras que tengan una mayor diferencia posible para así obtener un espacio semántico representativo. El Componente uno está mejor representado por los Kansei: "Innovador", "Listo para comer", "Nutritivo", "Bueno", "Brinda confianza", "Lo consumiría otra vez", "Placentero", "Emocionante", "Calidad" y "Me gusta". El Componente dos está representado por los Kansei "Peculiar" y "Llamativo" en función al lado positivo y con los Kansei "Clásico", "Natural" y "Tradicional" en función al lado negativo.En la Figura 4 se observa que las marcas de hot-dog: Razzeto, Otto Kunz y Braedt están posicionadas en distintos cuadrantes (segundo, tercero y cuarto, respectivamente) esto debido a lo mencionado anteriormente. El Componente uno está mejor representado por los Kansei: "Sabor intenso", "Sabor a pollo", "Oscuro", "Ahumado", "Textura externa dura", "Textura uniforme", "Duro", "Sabor definido", "Salado" y "Tamaño adecuado". El componente dos está mejor representado con los Kansei: "Con colorante", "Color uniforme", "Colorido", "Brilloso", "Condimentado", "Grumoso", "Olor fuerte" y "Aroma duradero".
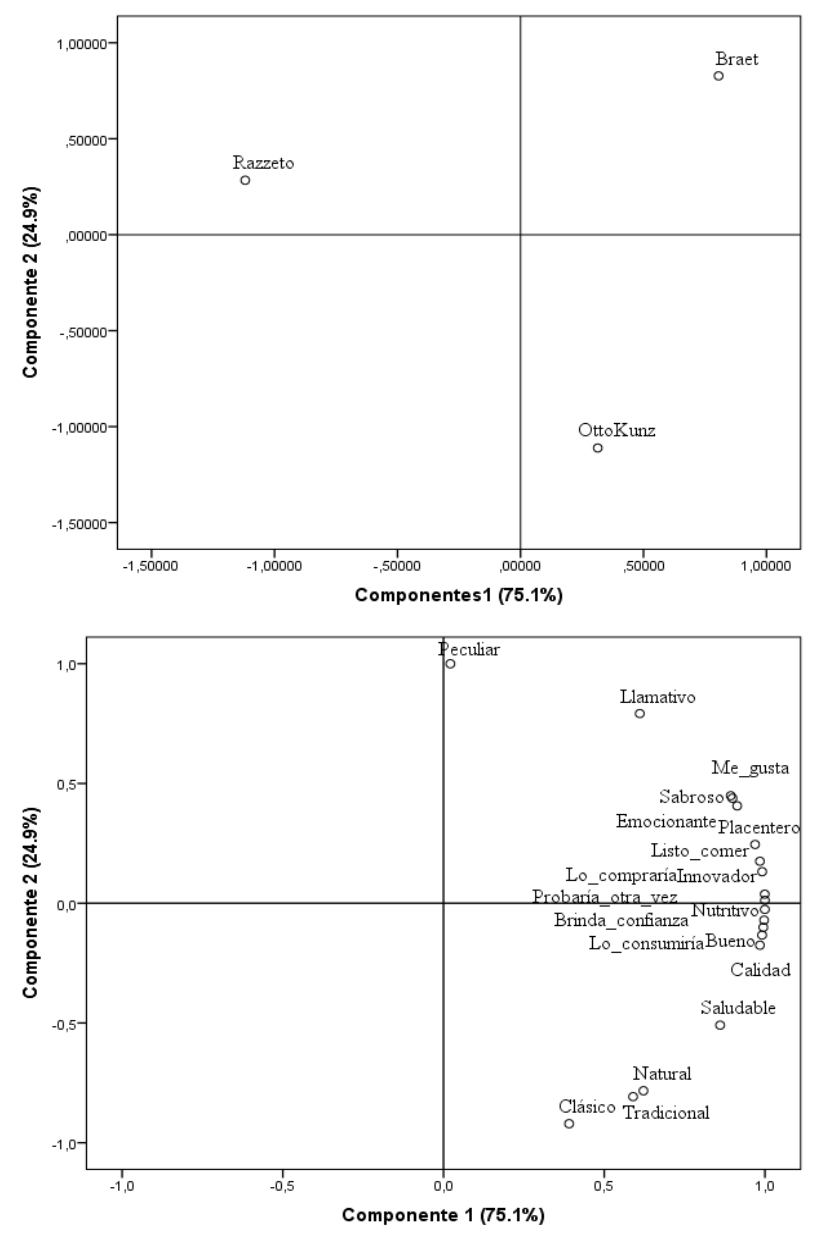

Figura 3. Representación de las tres marcas y los 19 atributos hedónicos evaluados en la primera y segunda dimensión del ACP 

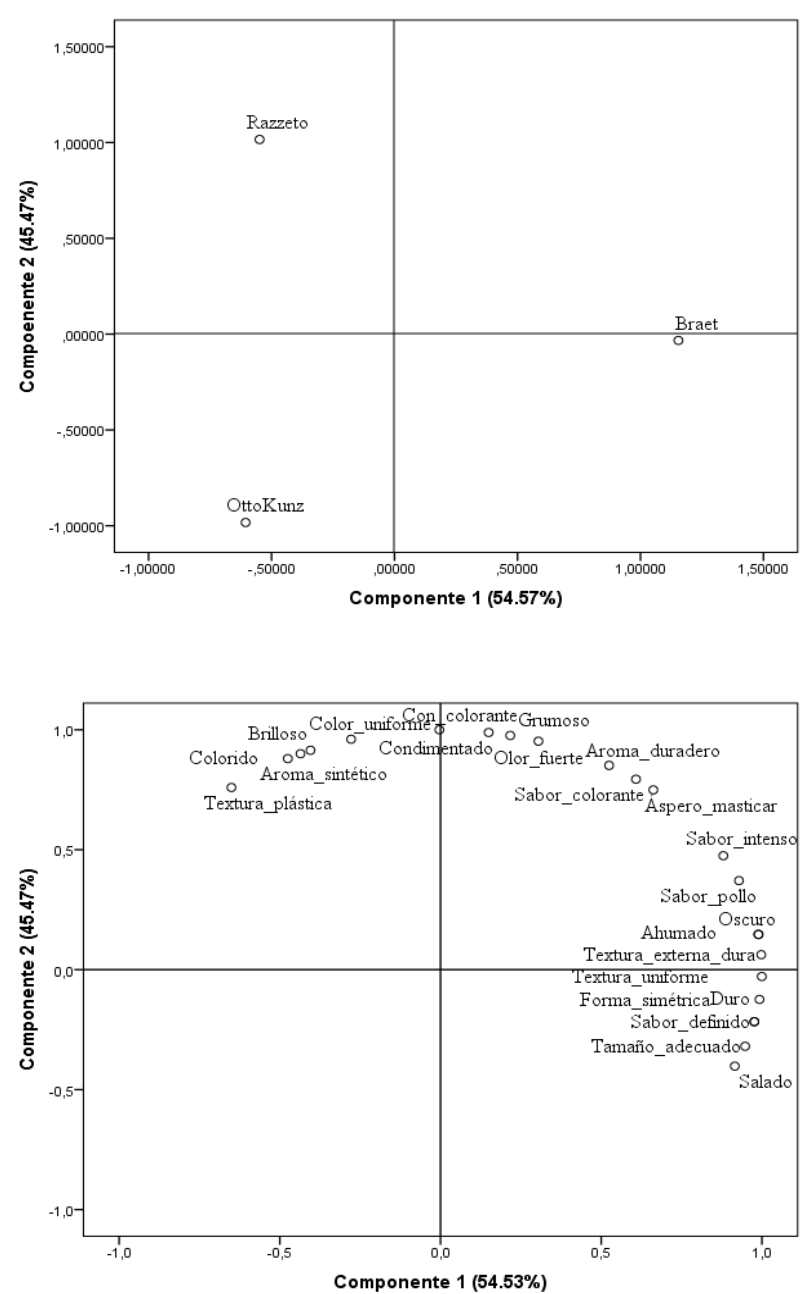

Figura 4. Representación de las tres marcas y los 23 elementos sensoriales evaluados en la primera y segunda dimensión de PCA

Marco-Almagro (2011) menciona que el análisis de componentes principales aplicado después del análisis clúster de k-medias en estudios de ingeniería Kansei es más bien una manera de comprobar la validez de los grupos obtenidos, dónde las palabras de un mismo clúster deben estar ubicados cerca en la gráfica puntos-variable.

Luego de realizar el análisis mixto, se obtuvo la base de datos kansei final para hot-dog a base de carne de pollo (Tabla 3) que está comprendida por ocho elementos sensoriales (cuatro de sabor, dos de color y dos de textura) y siete expresiones hedónicas, haciendo un total de 15 Kansei. Marco-Almagro (2011) en su investigación con jugos obtuvo 14 Kansei. Marco-Almagro y Schütte (2014) utilizaron 12 Kansei (cinco elementos sensoriales y siete expresiones hedónicas) para desarrollar barras de chocolate que den la impresión de ser deportivo y refrescante. En otro estudio con cañitas de carne los mismos autores utilizaron 13 Kansei (cinco elementos sensoriales y ocho expresiones hedónicas).

\section{Determinación del espacio de propiedades: Modelo Kano}

Los atributos se clasificaron como: unidimensionales, de encantamiento, neutros y obligatorios, en los cuadrantes I, II, III y IV, respectivamente (Llinares y Page, 2011). Como se puede observar en la Figura 5, las características de encantamiento fueron: funda comestible $(-0,2 ; 0,8)$, color $(-0,3 ; 0,7)$, tamaño $(-0,4 ; 0,7)$ y aspecto $(-0,2 ; 0,7)$, ya que tienen un Coeficiente de Satisfacción $(\mathrm{CS})>$ 0,50 y Coeficiente de Insatisfacción (CI) mayor a - 0,50 (Tontini, 2007; Matzler et al., 2004), por lo cual si dichas características estuvieran presentes en el producto van a aumentar la satisfacción del cliente, pero si no estuvieran no generan insatisfacción ( $\mathrm{CI}>-0,50)$; las características: sabor $(-0,9 ; 0,2)$, olor $(-0,9 ; 0,1)$, contenido de sal $(-0,6$; $0,1)$ y masticabilidad $(-0,6 ; 0,4)$ resultaron obligatorias; es decir, si las características estuvieran presentes no generarán mayor satisfacción, por el contrario, si no estuvieran presentes generarán insatisfacción; la característica: ahumado $(-0,4 ; 0,3)$ resultó neutra o no significativa.

No se encontró información de la aplicación del modelo Kano en la industria de alimentos; sin embargo, es una herramienta potencial para estudios de Ingeniería Kansei (Köhler et al., 2014; Liu et al., 2014).

Se eligieron dos características de encantamiento del modelo Kano para establecer el espacio de propiedades del hot-dog (Tabla 4). Las categorías evaluadas fueron carmín al 0,5 y 1,0\% para el color, y funda no comestible y comestible para el tipo de funda.

Tabla 3. Base de datos Kansei final para hot-dog a base de carne de pollo

\begin{tabular}{cc}
\hline Elemento sensorial & Expresión hedónica \\
\hline Color Uniforme & Tradicional \\
Forma simétrica & Natural \\
Sabor a pollo & Nutritivo \\
Brilloso & Llamativo \\
Condimentado & Innovador \\
Grumoso & Lo compraría \\
Ahumado & Me gusta \\
Salado & \\
\hline
\end{tabular}

Tabla 4. Categorías del espacio de propiedades

\begin{tabular}{ll}
\hline Elemento: color & \multicolumn{1}{c}{ Elemento: funda } \\
\hline 1: Carmín al $0,5 \%$ & 1: Funda no comestible \\
2: Carmín al $1,0 \%$ & 2: Funda comestible \\
\hline
\end{tabular}

\section{Síntesis}

En la Tabla 5 se muestran los resultados de la regresión QT1, donde se relacionaron cada una de las expresiones hedónicas (tradicional, natural, nutritivo, llamativo, innovador, lo compraría y me gusta) con las categorías de los elementos del espacio de propiedades (carmín al 0,5\%, carmín al 1,0\%, funda de celulosa y funda comestible). 


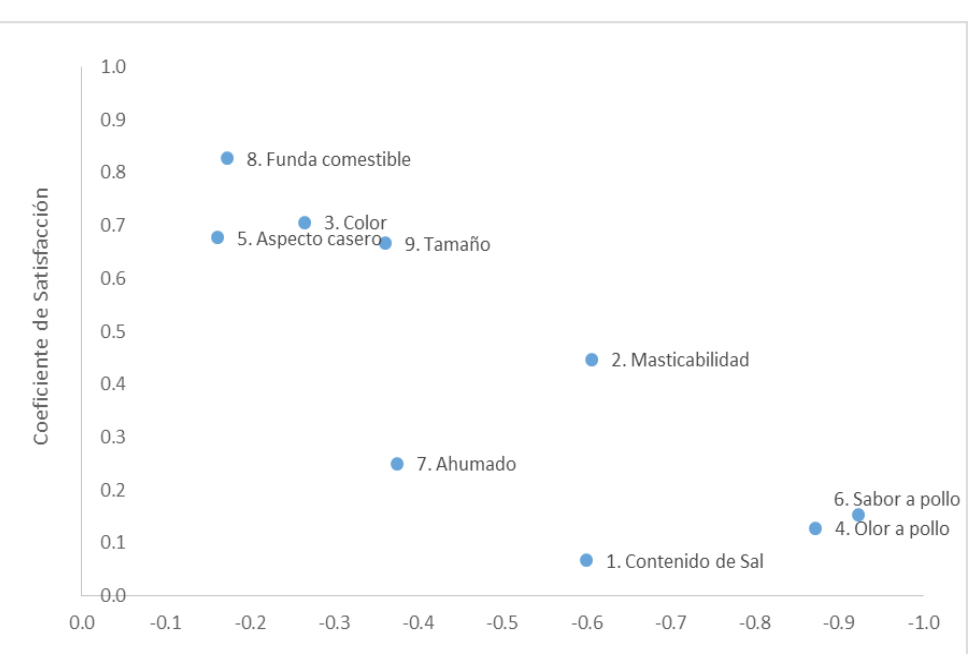

Coeficiente de Insatisfacción

Figura 5. Representación de las nueve características mediante el CS y CI de Kano

Tabla 5. Puntaje de Categoría (CP) y Coeficiente de Correlación Parcial (CCP) del análisis QT1 (expresiones hedónicas con espacio de propiedades)

\begin{tabular}{|c|c|c|c|c|}
\hline Kansei & Elemento & $\mathrm{CCP}$ & Categoría & $\mathrm{CP}$ \\
\hline \multirow{4}{*}{ Tradicional } & \multirow{2}{*}{ Color } & \multirow{2}{*}{0,359} & Carmín al 1,0\% & $-0,050$ \\
\hline & & & Carmín al 0,5 \% & 0,050 \\
\hline & \multirow{2}{*}{ Funda } & \multirow{2}{*}{0,794} & No comestible & $-0,170$ \\
\hline & & & Comestible & 0,170 \\
\hline \multirow{4}{*}{ Natural } & \multirow{2}{*}{ Color } & \multirow{2}{*}{0,972} & Carmín al 1,0 \% & 0,105 \\
\hline & & & Carmín al 0,5 \% & $-0,105$ \\
\hline & \multirow{2}{*}{ Funda } & \multirow{2}{*}{0,997} & No comestible & $-0,325$ \\
\hline & & & Comestible & 0,325 \\
\hline \multirow{4}{*}{ Nutritivo } & \multirow{2}{*}{ Color } & \multirow{2}{*}{0,600} & Carmín al 1,0\% & 0,060 \\
\hline & & & Carmín al 0,5 \% & $-0,060$ \\
\hline & \multirow{2}{*}{ Funda } & \multirow{2}{*}{0,832} & No comestible & $-0,120$ \\
\hline & & & Comestible & 0,120 \\
\hline \multirow{4}{*}{ Llamativo } & \multirow{2}{*}{ Color } & \multirow{2}{*}{0,910} & Carmín al 1,0\% & $-0,110$ \\
\hline & & & Carmín al 0,5 \% & 0,110 \\
\hline & \multirow{2}{*}{ Funda } & \multirow{2}{*}{0,994} & No comestible & $-0,450$ \\
\hline & & & Comestible & 0,450 \\
\hline \multirow{4}{*}{ Innovador } & \multirow{2}{*}{ Color } & \multirow{2}{*}{0,316} & Carmín al 1,0 \% & $-0,020$ \\
\hline & & & Carmín al 0,5 \% & 0,020 \\
\hline & \multirow{2}{*}{ Funda } & \multirow{2}{*}{0,992} & No comestible & $-0,480$ \\
\hline & & & Comestible & 0,480 \\
\hline \multirow{4}{*}{$\begin{array}{l}\text { Lo } \\
\text { compraría }\end{array}$} & \multirow{2}{*}{ Color } & \multirow{2}{*}{0,933} & Carmín al 1,0 \% & $-0,130$ \\
\hline & & & Carmín al 0,5 \% & 0,130 \\
\hline & \multirow{2}{*}{ Funda } & \multirow{2}{*}{0,997} & No comestible & $-0,630$ \\
\hline & & & Comestible & 0,630 \\
\hline \multirow{4}{*}{ Me gusta } & \multirow{2}{*}{ Color } & 0925 & Carmín al 1,0\% & $-0,170$ \\
\hline & & & Carmín al 0,5\% & 0,170 \\
\hline & Fund & 0994 & No comestible & $-0,650$ \\
\hline & Funda & 0,994 & Comestible & 0,650 \\
\hline
\end{tabular}

Los valores de CP y CCP expresan la influencia de cada categoría y cada elemento evaluada para un determinado Kansei, respectivamente (Nagamachi, 2011). En relación al Kansei "Tradicional", el color no tiene efecto significativo
$(\mathrm{CPP}=0,359)$, en contraste, el tipo de funda está más relacionado $(\mathrm{CPP}=0,794)$, observando que si el hot-dog tiene funda de colágeno $(\mathrm{CP}=0,170)$ el consumidor lo va a percibir como "Tradicional". Con relación al Kansei "Natural" ambos elementos (color y funda) tienen relación con dicho Kansei ( $\mathrm{CPP}=0,972$ y 0,997 ; respectivamente) y los $\mathrm{CP}$ que tienen valores positivo son: carmín al $1,0 \%$ y funda de colágeno; es decir, para que un hot-dog de la impresión de "Natural" tiene que tener 1,0\% de colorante carmín y funda de colágeno. Para el caso del Kansei "Nutritivo" la funda $(\mathrm{CPP}=0,832)$ tiene mayor relación que el color $(\mathrm{CPP}=0,600)$; por lo que para que el hot-dog de la impresión de "Nutritivo" tiene que tener $1,0 \%$ de colorante carmín y funda de colágeno ya que estos valores fueron positivos. Con relación al Kansei "Llamativo", el tipo de funda $(\mathrm{CPP}=0,994)$ tiene mayor relación que el color $(\mathrm{CPP}=0,991)$ por lo que para que el hot-dog, de la impresión de "Llamativo" tiene que tener colorante carmín al 0,5\% y ser de funda de colágeno. Con relación al Kansei "Innovador", el elemento funda (CPP $=0,992)$ tiene mayor influencia que el color (CPP = 0,316). Para el Kansei "Lo compraría", la funda tiene mayor influencia $(\mathrm{CPP}=0,997)$ que el color $(\mathrm{CPP}=0,933)$; sin embargo ambos elementos están relacionados con dicho Kansei. Para que el hotdog genere el Kansei "Lo compraría" debe tener 0,5\% de colorante carmín y funda comestible. Por último, para el Kansei "Me gusta" la funda tiene mayor influencia (CPP $=0,994)$ que el color $(\mathrm{CPP}=0,925)$. Para que el hot-dog genere el Kansei "Me gusta" debe estar formulado con $0,5 \%$ de colorante carmín y debe tener funda de colágeno.

En la Tabla 6 se muestran los resultados de la regresión QT1, en la cual se relacionaron cada uno de los Kansei (atributos sensoriales: color uniforme, forma simétrica, sabor a pollo, brilloso, condimentado, grumoso, ahumado y salado) con las categorías de los elementos del espacio de propiedades (carmín al 0,5\%, carmín al 1,0\%, funda de celulosa y funda comestible).

Los Kansei: "Color uniforme", "Forma simétrica", "Brilloso", "Grumoso" y "Ahumado", tienen la misma tendencia respecto a las características: carmín al 0,5\% y funda de colágeno, las cuales si están presentes en el producto van a provocar que el consumidor perciba dichos Kansei. Para el caso del Kansei "Grumoso", la funda tiene efecto predominante sobre el Kansei $(\mathrm{CPP}=0,600)$ a diferencia del color $(\mathrm{CPP}=0,385)$.

Los Kansei "Sabor a pollo", "Condimentado" y "Salado", tienen la misma tendencia respecto a las características: carmín al 1,0\% y funda de celulosa, las cuales si están presentes en el producto van a provocar que el consumidor perciba dichos Kansei. Para el caso del Kansei "Condimentado", la funda tiene efecto predominante sobre el Kansei $(\mathrm{CPP}=0,863)$ a diferencia del color $(\mathrm{CPP}=0,173)$; lo contrario ocurre con el Kansei 
"Salado", donde el color $(\mathrm{CPP}=0,732)$ tiene efecto predominante sobre el Kansei a diferencia del elemento funda $(\mathrm{CPP}=0,377)$.

Son pocas las investigaciones que se han realizado sobre Ingeniería Kansei en alimentos; la mayoría de estudios están realizados en productos como lápices, refrigeradores, polos (Nagamachi y Mohd, 2011) y envases de cerveza (Nagamachi, 2011; Hirata, 2009).

Tabla 6. Puntaje de Categoría (CP) y Coeficiente de Correlación Parcial (CCP) del análisis QT1 (elementos sensoriales con espacio de propiedades)

\begin{tabular}{|c|c|c|c|c|}
\hline Kansei & Elemento & $\mathrm{CCP}$ & Categoría & $\mathrm{CP}$ \\
\hline \multirow{4}{*}{ Color uniforme } & \multirow{2}{*}{ Color } & \multirow{2}{*}{0,931} & Carmín al 1,0\% & $-0,230$ \\
\hline & & & Carmín al 0,5\% & 0,230 \\
\hline & \multirow{2}{*}{ Funda } & \multirow{2}{*}{0,984} & No comestible & $-0,490$ \\
\hline & & & Comestible & 0,490 \\
\hline \multirow{4}{*}{ Forma simétrica } & \multirow{2}{*}{ Color } & \multirow{2}{*}{0,931} & Carmín al 1,0 \% & $-0,230$ \\
\hline & & & Carmín al 0,5\% & 0,230 \\
\hline & \multirow{2}{*}{ Funda } & \multirow{2}{*}{0,984} & No comestible & $-0,490$ \\
\hline & & & Comestible & 0,490 \\
\hline \multirow{4}{*}{ Sabor a pollo } & \multirow{2}{*}{ Color } & \multirow{2}{*}{0,999} & Carmín al 1,0\% & 0,190 \\
\hline & & & Carmín al 0,5\% & $-0,190$ \\
\hline & \multirow{2}{*}{ Funda } & \multirow{2}{*}{0,707} & No comestible & 0,010 \\
\hline & & & Comestible & $-0,010$ \\
\hline \multirow{4}{*}{ Brilloso } & \multirow{2}{*}{ Color } & \multirow{2}{*}{0,994} & Carmín al 1,0\% & $-0,023$ \\
\hline & & & Carmín al 0,5\% & 0,023 \\
\hline & \multirow{2}{*}{ Funda } & \multirow{2}{*}{0,999} & No comestible & $-0,603$ \\
\hline & & & Comestible & 0,603 \\
\hline \multirow{4}{*}{ Condimentado } & \multirow{2}{*}{ Color } & \multirow{2}{*}{0,173} & Carmín al 1,0\% & 0,030 \\
\hline & & & Carmín al 0,5\% & $-0,030$ \\
\hline & \multirow{2}{*}{ Funda } & \multirow{2}{*}{0,863} & No comestible & 0,290 \\
\hline & & & Comestible & $-0,290$ \\
\hline \multirow{4}{*}{ Grumoso } & \multirow{2}{*}{ Color } & \multirow{2}{*}{0,385} & Carmín al 1,0\% & $-0,100$ \\
\hline & & & Carmín al 0,5\% & 0,100 \\
\hline & \multirow{2}{*}{ Funda } & \multirow{2}{*}{0,600} & No comestible & $-0,180$ \\
\hline & & & Comestible & 0,180 \\
\hline & Color & 0707 & Carmín al 1,0\% & $-0,030$ \\
\hline Ahumado & Color & $0, / 01$ & Carmín al 0,5\% & 0,030 \\
\hline Anumado & Funda & 0857 & No comestible & $-0,050$ \\
\hline & Funda & 0,85 & Comestible & 0,050 \\
\hline & Color & 0732 & Carmín al 1,0\% & 0,290 \\
\hline Salado & Color & 0,132 & Carmín al 0,5\% & $-0,290$ \\
\hline salado & & & No comestible & 0,110 \\
\hline & Func & $0,3 / 1$ & Comestible & $-0,110$ \\
\hline
\end{tabular}

Prithiviraj y Grzechnik (2010) evaluaron en nueve prototipos de wafer, utilizando el Software KESo con 20 consumidores, el efecto de la cantidad de chocolate $(1 \mathrm{~mm}$, $2 \mathrm{~mm}$ y $3 \mathrm{~mm}$ de espesor) y cantidad de obleas (1, 2 y 3 obleas), sobre cinco Kansei (preferible, estilo de vida, gratificante, de calidad e inferior) obteniendo valores de PPC entre 0,50 a 0,91 ; en donde concluyeron que los wafer en estudio son preferibles, gratificantes y de calidad si poseen más contenido de chocolate y moderadamente cantidad de obleas. La experiencia emocional entre los productos con más cantidad de chocolate y menos número de obleas se percibe como un estilo de vida, y con menos cantidad de chocolate y un gran número de obleas se percibe como inferior. Además, Zhao (2010) evaluó en ocho prototipos el efecto de la cantidad de azúcar, mantequilla y levadura en polvo en cinco Kansei (tentador, delicado, crujiente, disfrutar y juvenil), concluyendo que para el Kansei tentador, ninguno de los tres elementos tiene efecto significativo; sin embargo el más importante es el azúcar, resultando que una menor cantidad de esta, aumenta dicho Kansei. Para el caso del Kansei delicado, el azúcar tuvo más influencia, comportándose de igual manera que el Kansei anterior.

\section{Conclusiones}

Se aplicó y explicó una metodología capaz de integrar las necesidades afectivas del consumidor en productos alimenticios. La metodología se basa en la Ingeniería Kansei (IK), que comprende las etapas: elección del dominio, generación del espacio semántico, generación del espacio de propiedades y síntesis. Se demostró que es factible la traducción de necesidades emocionales en elementos de diseño a través de la IK tipo II. El modelo Kano fue relevante para determinar las características emocionales que atraen al consumidor, las cuales se incorporaron en los prototipos de hot-dog. La IK, permite tener información cuantitativa del efecto que generan las características de un producto en la satisfacción emocional del consumidor. Se validó el uso del método mixto que combina una etapa cualitativa (Diagrama de Afinidad) y otra cuantitativa (Análisis Clúster de k-medias y Análisis de Componentes Principales), para la obtención de las necesidades Kansei de hot-dog de carne de pollo del sector socioeconómico B y C de Lima Metropolitana, obteniéndose 15 Kansei (ocho elementos sensoriales: cuatro de sabor, dos de color y dos de textura; y siete expresiones hedónicas). El prototipo de hot-dog que satisface los Kansei: "Tradicional", "Innovador", "Lo compraría", "Llamativo", "Me gusta", "Color uniforme", "Forma simétrica", "Brilloso" y "Ahumado", está determinado por los elementos: carmín al 0,5 por ciento y funda comestible.

\section{Literatura citada}

Álvarez, H. 2009. Metodología para el Desarrollo de Productos Alimentarios con Alto Contenido Emocional (Kansei Engineering). Editorial Apsoluti S.L. Barcelona, España.

Álvarez, H. 2011. Cómo Diseñar y Desarrollar Productos con Alto Contenido Emocional a través de la Ingeniería Kansei. Editorial Apsoluti S.L. Barcelona, España.

APEIM, 2016. Niveles Socioeconómicos. Disponible en: http://www.apeim.com.pe/wp-content/themes/apeim/ docs/nse/APEIM-NSE-2016.pdf. Consultado el 10-0317.

Damodar, G. 2005. Econometría. Trad. Garmendía, D. Cuarta edición. Editorial Mc Graw-Hill. México D.F., México. 
Elías, C.; Chirinos, R. y Salvá, B. 2000. Elaboración de embutidos. Lima, Perú, s.e. 91 p.

Ezequiel, U. y Aldas, J. 2005. Análisis Multivariante Aplicado: Aplicaciones al Marketing, investigación de mercados, economía, dirección de empresa y turismo. Editorial Paraninfo S.A. Madrid, España.

Hirata, R. 2009. Traducción de las Emociones y Sensaciones del Cliente en Productos y Servicios: Una Herramienta de la Sexta Generación de Calidad. Tesis Doctoral. Universidad Nacional Autónoma de México. México D.F., México.

Johnson, R. y Wichern, D. 2007. Applied multivariate statistical analysis. Editorial Pearson Education. Londres, Inglaterra

Köhler, M.; Falk, B. y Schmitt, R. 2014. Applying EyeTracking In Kansei Engineering Methodology For Design Evaluations In Product Development. International Conference On Kansei Engineering And Emotion Research (pp. 837-852). Linköping, Suecia.

Liu, P.; Lin, S.; Wang, C. y Chuang, M. 2014. The kitchen supply design based on Kansei engineering study for the elderly user of independent. International Conference On Kansei Engineering And Emotion Research (pp. 959 - 967). Linköping, Suecia.

Llinares, C. y Page, A. 2011. Kano's model in Kansei Engineering to evaluate subjetive real estate consumer preferences. Int. Journal of Industrial Ergonomics 41 (3): 233-246.

Marco-Almagro, L. 2011. Statistical Methods in Kansei Engineering Studies (Tesis Doctoral). Universidad Politécnica de Catalunya. Barcelona, España.

Marco-Almagro, L. y Schütte, S. 2014. Development of an Affective Sensorial Analysis Method for the Food Industry. International Conference on Kansei Engineering and Emotion Research. Linköping, Suecia. 23 p.

Matzler, K.; Fuchs, M. y Schubert, A. 2004. Employee Satisfaction: Does Kano's Model Apply?. Total Quality Management \& Business Excellence 15(9-10): 11791198.

Meullenet, J.; Xiong, R. y Findlay, C. 2007. Multivariate and Probabilistic Analyses of Sensory Science Problems. IFT Press. Editorial Advisory Board. Iowa, Estados Unidos.

Nagamachi, M. 1994. Kansei Engineering: An ergonomic technology for a product development. Journal of Proceedings of IEA 94: 1220-122.

Nagamachi, M. 2011. Kansei/Affective Engineering. Editorial CRC Press y Taylor \& Francis Group. Florida, Estados Unidos.

Nagamachi, M.; Mohd, A. 2011. Innovations of Kansei engineering. Editorial CRC Press y Taylor \& Francis Group. Florida, Estados Unidos.
Palazuelos, J. y Blázquez, O. 2013. El Mercado de Embutidos y Jamón en Perú. ICEX-España Exportación e Importación. Editorial de la Oficina Económica y Comercial de la Embajada de España en Lima. Lima, Perú.

Prithiviraj, A. y Grzechnik, D. 2010. The Influence of Chocolate and Wafer on customers - An Application of Kansei Engineering.Degree Project. Department of Management and Engineering. Linköping, Suecia.

Puma, G. 2017. Aplicación de la metodología Kansei tipo II para desarrollo de hot-dog a base de carne de pollo (Gallus gallus) (Tesis para optar el título de ingeniero en Industrias Alimentarias). Facultad de Industrias Alimentarias, Universidad Nacional Agraria La Molina. Lima, Perú.

Schütte, S. 2002. Designing Feelings into Products: Integrating Kansei Engineering Methodology in Product Development. (Tesis $\mathrm{N}^{\circ}$ 946). Linköping Studies in Science and Technology. Linköping, Suecia

Schütte, S. 2005. Engineering Emotional Values in Product Design: Kansei Engineering in Development.(Thesis $\mathrm{PhD}$ ). Linköping Studies in Science and Technology. Linköpings Universitet. Linköping, Suecia

Tontini, G. 2007. Integrating the Kano Model and QFD for Designing New Products. Journal of Total Quality Management \& Business Excellence 18 (6): 599-612.

Varela, P. \& Ares, G. 2014. Novel Techniques in Sensory Characterization and Consumer Profiling. Editorial CRC Press y Taylor and Francois Group. Florida, Estados Unidos.

Zhao, Y. 2010. Developing a wafer with customer amenity - an application of Kansei engineering. Degree Project Department of Management and Engineering. LIU-IEI-TEK-A--09/00704—SE (53). Linköping, Suecia. 